\title{
Handwritten Artefact Identification Method for Table Interpretation with Little Use of Previous Knowledge
}

\author{
Luiz Antônio Pereira Neves ${ }^{1}$, João Marques de Carvalho ${ }^{1}$, Jacques Facon ${ }^{2}$, \\ Flávio Bortolozzi ${ }^{2}$, and Sérgio Aparecido Ignácio ${ }^{2}$ \\ 1 UFCG-Universidade Federal de Campina Grande, \\ Caixa Postal 105, 58.109-970, Campina Grande, Paraíba, Brasil \\ \{neves, carvalho\}adee.ufcg.edu.br \\ 2 PUCPR-Pontifícia Universidade Católica do Paraná, \\ Rua Imaculada Conceição 1155, \\ Prado Velho 80215-901, Curitiba-PR, Brazil \\ ffacon, fborto\}@ppgia.pucpr.br, \\ s.ignacio@pucpr.br
}

\begin{abstract}
An artefact identification method for handwritten filled table-forms is presented. Artefacts in table-forms are smudges and overlaps between handwritten data and line segments which increase the complexity of table-form interpretation. After reviewing some knowledge-based methods, a novel artefact identification method to improve table-form interpretation is presented. The proposed method aims to detect, identify and remove table-form artefacts with little use of previous knowledge. Experiments show the significance of using the proposed artefact identification method to improve table-form interpretation rates.
\end{abstract}

\section{Introduction}

Tables, or table-forms, are documents composed by cells, which are determined by intersections of straight line segments, as illustrated in figure 1

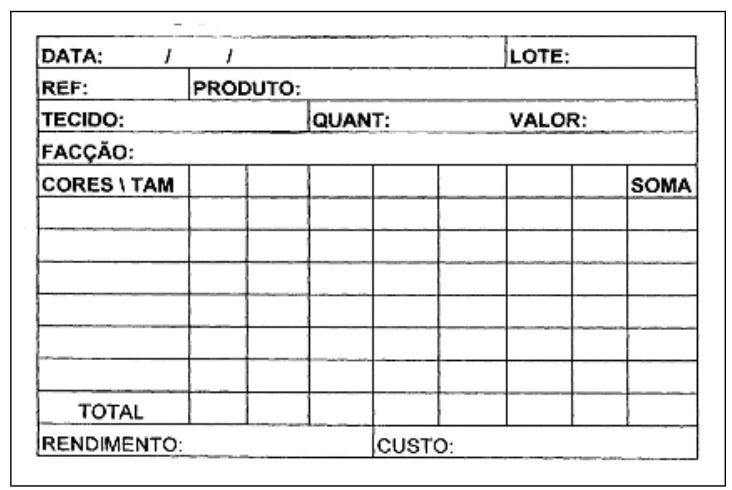

Fig. 1. Example of a table-form document

H. Bunke and A.L. Spitz (Eds.): DAS 2006, LNCS 3872, pp. 176-185 2006.

(C) Springer-Verlag Berlin Heidelberg 2006 
Several studies have been presented on table recognition [1], [2], [3], [4], [5], [6], [7], [8], [9]. Some of these researches use tables without imperfections in the horizontal and vertical line segments to reduce the complexity of the problem. In the damaged table case, many researchers use previous knowledge for their interpretations, also aiming to minimize the complexity of the problem.

Since the table-forms considered in this work can be filled in by machines or by hand, overlaps between printed and handwritten information, as shown in figures 2 -a and2 2 b, might create false intersections. These occurrences are called artefacts. Besides, artefacts can result from faulty table lines, from problems with document acquisition, from binarization problems, or simply from the poor quality of the document, as illustrated by the damaged piece of paper in figure2-c. Thus, an artefact can be defined as any object, handwritten or not, that produces false table-form intersections. Problems created by artefacts have been partially solved by other researchers. Watanabe [7],[8] presents two procedures, one to be used when no information is previously known and another one when artefacts (noise) characteristics are available in a knowledge base. Couasnon [3] uses previous noise and imperfections knowledge as grammar rules. Arias and Kasturi [1], [2] use the morphological closing operator to eliminate imperfections and to recover the extinguished segment lines for the analysis of the table-form intersections. Liang et al. [10] consider noise and imperfections as previous knowledge. Hori, Doermann [11] and Hirano et al. [12] use table models with noise and imperfections. Shinjo et al. [13] use previous knowledge to detect and correct damages of table corners. Shimotsuji and Asano [14] use table models with imperfections as previous knowledge, to be identified in the interpretation process. Pizano [15] performs image size reduction to eliminate noise and segments, combined with the use of minimum width and distance parameters to eliminate the remaining noise. Tran van Thom [6] performs image size reduction and thresholding for detecting and correcting segments with imperfections.

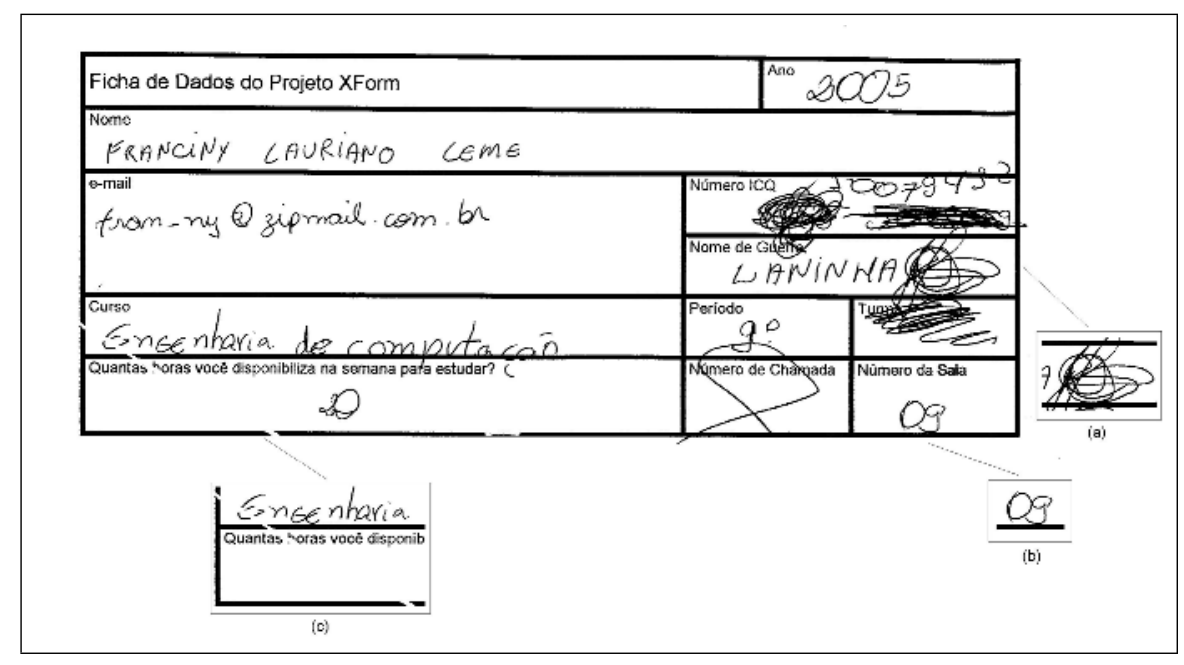

Fig. 2. Examples of artefacts 
Analyzing the solutions above, one can conclude that for all methodologies described some form of a priori knowledge is needed. The aim of this paper is to propose an artefact analysis method with very little use of a priori knowledge, to improve tableform interpretation. This method is described in Section 2 Section 3 presents some experimental results and discussions. Finally, the conclusions are given in Section 4

\section{Artefact Identification}

The proposed artefact identification method is based on compactness analysis. Compactness is a property that expresses how large is the area concentrated inside a given perimeter, as shown in figure 3 . Compactness is measured by the compactness factor, computed from the perimeter and the area of the analyzed shape. Given a shape of perimeter $P$ and area $S$, its compactness factor is given by $F C$, as shown in the equation 1 .

$$
F c=\frac{P^{2}}{4 \pi S}
$$

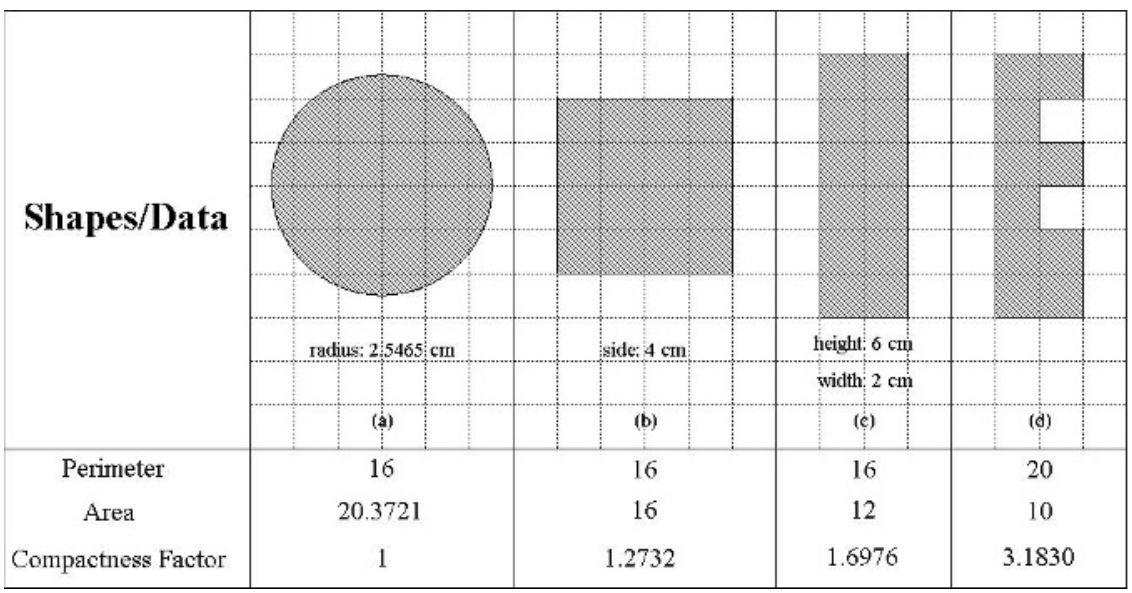

Fig. 3. Compactness Analysis for artefact identification

Verifying the shapes in the figure 3 , the circle (figure 3-a) presents the best compactness and we can say that, in general, table-form artefacts present high compactness, with values equal or around 1 . Thereby, a threshold has been created for distinguishing if the value calculated for the compactness factor corresponds to that of an artefact or to a straight line segment of a table cell. For determining threshold value, compactness factors from more than 30 different artefacts were submitted to exploratory data analysis [16], characterizing a homogenous distribution with a confidence level of $99 \%$. The range of variation $\mu \pm 2.576 * \sigma$, where $\mu$ and $\sigma$ are the mean and standard deviation respectively, produces inferior and superior limits of 1.21688 and 1.37419 , respectvely. The 
$0.5 \%$ of values above the superior limit are not contemplated as artefacts. Therefore, the handwritten data that presents compactness factor below 1.4 is considered an artefact.

Figures 4 - a and 4 -b show several types of artefacts with the respective compactness factors.

\begin{tabular}{|c|c|c|}
\hline index & artefact & $\begin{array}{c}\text { compactness } \\
\text { factor }\end{array}$ \\
\hline (a) & & 1.28998 \\
\hline (b) & & 1.27324 \\
\hline (c) & & 1.34486 \\
\hline (d) & & 1.28857 \\
\hline (e) & & 1.28547 \\
\hline (f) & & \\
\hline
\end{tabular}

(a)

\begin{tabular}{|c|c|c|}
\hline index & artefact & $\begin{array}{c}\text { compactness } \\
\text { factor }\end{array}$ \\
\hline (a) & L & 1.29841 \\
\hline (b) & & 1.28477 \\
\hline (c) & & 1.27404 \\
\hline (d) & & 1.28803 \\
\hline (e) & 1.29628 \\
\hline (f) & & 1.28137 \\
\hline
\end{tabular}

(b)

Fig. 4. Examples of Artefacts with their compactness factors

\begin{tabular}{c|c|c|} 
index & segment & $\begin{array}{c}\text { compactness } \\
\text { factor }\end{array}$ \\
\hline (a) & & 1.94365 \\
\hline (b) & & 4.98904 \\
\hline (c) & & 1.59781 \\
\hline (d) & & 5.27407 \\
\hline (e) & & 3.17814 \\
\hline (f) & & \\
\hline
\end{tabular}

Fig. 5. Examples of segments that are not artefacts with their compactness factors 
Figure 5 shows some table segments with compactness factor values above the established threshold. For figure 5-d, for instance, the compactness factor is 5.27407. This value indicates that the analyzed object is not an artefact, but rather a segment. Therefore, by observing figures 4 a, 4 $\mathrm{b}$ and 5 , one can conclude that the artefact identification method can make the correct distinction between a handwritten artefact and a table segment.

\subsection{Overlap Analysis Step}

Overlaps between handwritten data and segments produce misleading values of compactness factors. Analyzing the Pearson variation coefficient [16], [17], using data generated from the horizontal projection profile, it is possible to carry out their elimination. The detection of a peak, which indicates the presence of an undesirable segment among the data, means that an overlap should be deleted, as shown in figure 6-a. By using again the exploratory data analysis [16], one can define the sample variation range which permits elimination of possible undesirable overlaps (table 1). High variability of pixel density values in the horizontal direction (using a confidence level of $90 \%$ ), characterizes heterogeneous distributions. Then, the range of variation $\mu \pm 1.645 * \sigma$, where $\mu$ and $\sigma$ are the mean and standard deviation respectively, permits to delete the $5 \%$ of superior values out of this limit, resulting in overlap elimination, as depicted in figure 6 - b. Table 1 shows the data analysis for figure 6.a, where the variation coefficient is high, $74.58 \%$. Therefore, the high standard deviation indicates that the artefact of figure 6-a contains overlaps. Consequently, the overlaps (here the segments) are removed, as illustrated in figure 6. $b$.

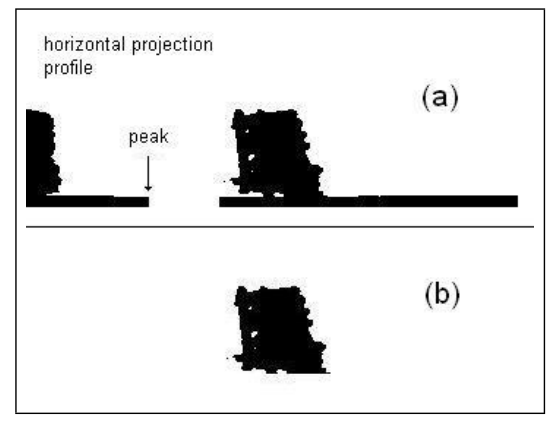

Fig. 6. Example of Artefact with overlapping

Table 1. Analysis of Pearson variation coefficient for figure 6

\begin{tabular}{|c|c|c|c|}
\hline Analysis & Data & Analysis & Data \\
\hline \hline Line Numbers & 67 & Inferior Limit & -15.0473 \\
Mean & 66.3284 & Superior Limit & 147.704 \\
Standard Deviation & 49.4685 & Variation Coefficient & 0.745812 \\
\hline
\end{tabular}




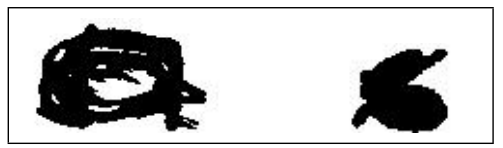

(a)

(b)

Fig. 7. Examples of analyzed handwritten data, processed without alteration

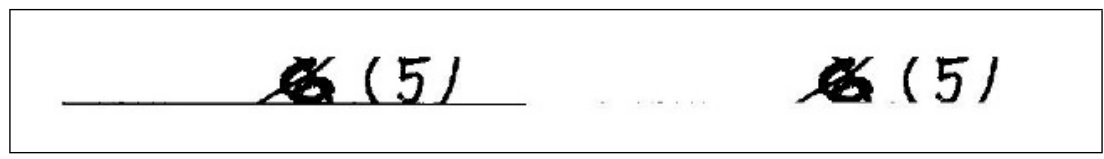

(a)

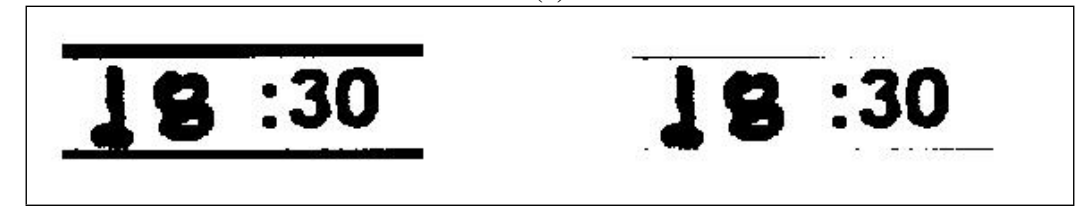

(b)

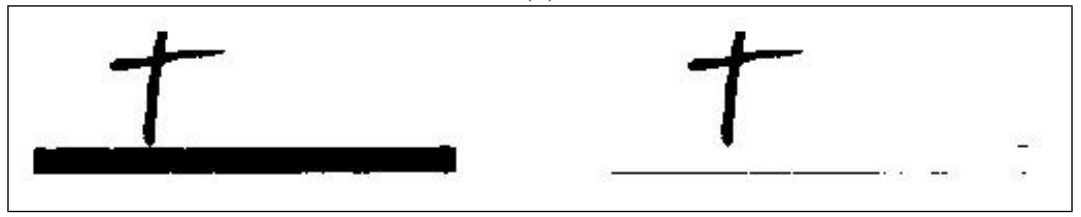

(c)

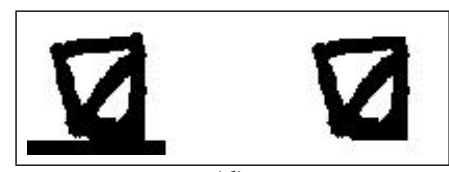

(d)

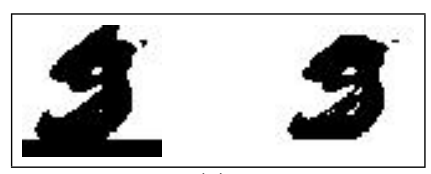

(e)

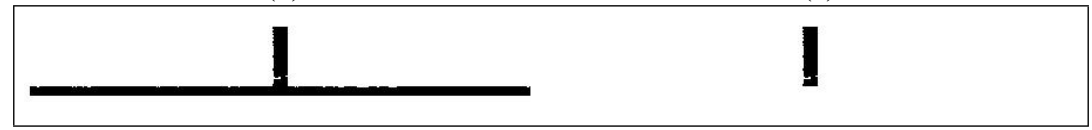

(f)
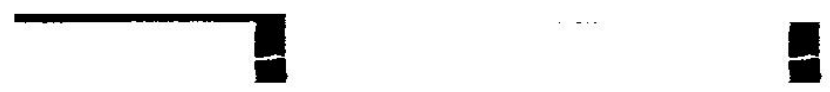

(g)

Fig. 8. Examples of artefacts with overlap

Figures 7 and 8 depict some results of artefact analysis and overlap removal. Figures 7 $\mathrm{r}$ a and 7.b show two successful examples of isolated artefacts without overlap, which represent valid information that must not be removed. Artefact analysis has indicated that the corresponding distributions are homogeneous, since the variation coefficients of figures 77 a and 7-b are $22.96 \%$ and $29.32 \%$ respectively. So they are not removed. 
Figure 8 -a shows four overlaps between handwritten data and table lines. These overlaps produce incorrect compactness coefficients. The same occurs in figures 8 -b and 8-c. By removing these overlaps, it is possible to correctly identify the true handwritten data. Similarly, overlap analysis makes correct artefact identification easier for figures 8-d and 8-e. Examples of artefacts due to failure in line segments are exhibited in figures 8 f and $8 \mathrm{~g}$, for which overlaps have been correctly eliminated.

\subsection{Artefact Cases}

Figures 9-a and 9-b illustrate cases where the artefact analysis method does not perform correct artefact identification. This happens because the handwritten letter $f$ (figure 9 . a), as well as the handwritten digits $l$ (figure 9-b) are similar to the table lines. The resulting shapes produce high compactness factors and the method does not consider them as artefacts. These cases represent challenges that will be the subject of further studies.

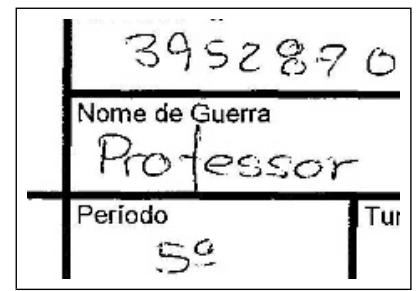

(a)

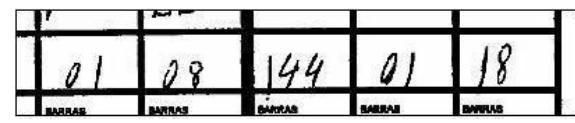

(b)

Fig. 9. Cases of table with artefact

\section{Experimental Results and Analysis}

To evaluate the performance of the proposed artefact identification approach, 305 tableform images were used to compose the test database, as exemplified in figures 10, 11 and 12 These table-form images, scanned at $300 \mathrm{dpi}$, are filled with handwritten data, handwritten overlap, and contain artefacts.

The table-form interpretation method used in the experiments has been previously described by this author [18]. The three fundamental steps are:

Step 1. Identification of table-form intersections;

Step 2. Corner detection and correction;

Step 3. Table interpretation and cell extraction.

The proposed artefact identification approach is inserted between Steps 2 and 3. Tests were carried out with and without artefact analysis in order to quantify the improvement produced by the proposed approach. The rate of processed images, shown in table 2 , indicates the percentage of images that went through all steps of the methodology. Rejected images are those that did not reach the final processing stage of the methodology. Correctly interpreted images are images that presented no interpretation 
Table 2. Summarized results of tests with 350 images

\begin{tabular}{llll} 
Method & $\begin{array}{l}\text { Rate of } \\
\text { processed } \\
\text { images }\end{array}$ & $\begin{array}{l}\text { Rate of } \\
\text { rejected } \\
\text { images }\end{array}$ & $\begin{array}{l}\text { Rate of correctly } \\
\text { interpreted } \\
\text { images }\end{array}$ \\
\hline Without using artefact analysis & $211(69 \%)$ & $94(31 \%)$ & $196(64 \%)$ \\
With using artefact analysis & $299(98 \%)$ & $6(2 \%)$ & $260(85 \%)$ \\
\hline
\end{tabular}

errors, i.e., their contents were $100 \%$ correctly interpreted. Initially, with no artefact analysis, 211 images (69\%), were correctly processed and 94 images (31\%) were rejected. From the 211 correctly processed images, 196 (64\%) were correctly interpreted. The process was then repeated applying artefact analysis. 299 images (98\%) were correctly processed and 6 images (2\%) were rejected. For the 299 processed images, 260 $(85 \%)$ were correctly interpreted. A significant result that can be observed is that without artefact analysis $31 \%$ of the table-form images in the base were rejected, whereas this index decreased to $2 \%$, keeping an index of $85 \%$ for correctly interpreted images, by applying artefact analysis. These results are summarized in table 2

\section{Conclusions}

An original identification method, for detecting and deleting smudges and overlaps between data and faulty segments in handwritten filled table-forms has been proposed in this paper. Based on the variation interval $\mu \pm 1.645 * \sigma$, on the coefficient of Pearson and on the compactness property, the proposed artefact identification method has shown to be effective in identifying different kinds of artefacts and in deleting overlaps. The experimental results presented show the significance of using the proposed artefact identification method to improve table-form interpretation rates.

Summarizing the advantages of the method, we mention the possibility of applying it to different types of handwritten filled table-forms for identification of handwritten smudges, of overlaps between handwritten data and table lines, as well as the intersection defects, all that with very little use of a priori knowledge.

\section{Acknowledgments}

We would like to acknowledge support for this research from UFCG, PUCPR and the PROCAD Program from CAPES/MEC (Brazilian government, project number 153/01-1).

\section{References}

1. Arias, J.F., Chhabra, A., Misra, V.: Finding Straight Lines in Drawings. ICDAR 1997 IEEE In: Proceedings of the Fourth International Conference on Document Analysis and Recognition (1997) 788-791

2. Arias, J.F., Kasturi, R., Chhabra, A.: Efficient Techniques for Telephone Company Line Drawing Interpretation. ICDAR 1995 - IEEE - In: Proceedings of the Third International Conference on Document Analysis and Recognition (1995) 795-798 
3. Couasnon, B.: Dmos: A generic document recognition method, application to an automatic generator of musical scores, mathematical formulae and table structures recognition systems. ICDAR 2001 - In: Proceedings of the Sixth International Conference on Document Analysis and Recognition (2001) 215-220

4. Fan, K.C., Lu, J.M., Wang, L.S., Liao, H.Y.: Extraction of characters from form documents by feature point clustering. Pattern Recognition Letters (1995)

5. Hu, J., Kashi, R.S., Lopresti, D., Wilfong, G.T.: Evaluating the performance of table processing algorithms. International Journal on Document Analysis and Recognition 4 (2002) 140 153

6. Thom, R.T.V.: Modélisation de Tableaux pour le traitement Automatique des Formulaires. Laboratoire PSI, Université de Rouen (1997)

7. Watanabe, T., Luo, Q., Sugie, N.: Structure recognition methods for various types of documents. Machine Vision and Applications (1993)

8. Watanabe, T., Luo, Q., Sugie, N.: Layout recognition of multi-kinds of table-form documents. IEEE Transactions on Pattern Analysis and Machine Intelligence (1995)

9. Kieninger, T., Dengel, A.: The t-recs table recognition and analysis system. In: DAS'98 Proceedings of the Sixth International Conference on Document Analysis Systems (1998) 255-269

10. Liang, J., Ha, J., Haralick, R.M., Phillips, I.T.: Document layout structure extraction using bounding boxes of different entities. WACV 1996 In: Proceedings of the Third IEEE Workshop on Applications of Computer Vision (1996) 278-283

11. Hori, O., Doermann, D.S.: Robust table-form structure analysis based on box-driven reasoning. ICDAR 1995 - In: Proceedings of the Third International Conference on Document Analysis and Recognition (1995) 218-221

12. Hirano, T., Okada, Y., Yoda, F.: Field extraction method from existing forms transmitted by facsimile. ICDAR 2001 - In: Proceedings of the Sixth International Conference on Document Analysis and Recognition (2001) 738-742

13. Shinjo, H., Hadano, E., Marukawa, K., Shima, Y., Sako, H.: A recursive analysis for form cell recognition. ICDAR 2001 In: Proceedings of the Sixth International Conference on Document Analysis and Recognition (2001)

14. Shimotsuji, S., Asano, M.: Form Identification based on Cell Structure. ICPR 1996 - IEEE - In: Proceedings of the 12th IAPR International Conference on Pattern Recognition (1996) 793-797

15. Pizano, A.: Extracting line features from images of business forms and tables. IAPR - In: Proceedings of the 11th International Conference on Pattern Recognition 3 (1992) 399-403

16. Tukey, J.W.: Exploratory Data Analysis. Addison-Wesley (1977)

17. Kazmier, L.J.: Estatística Aplicada a Economia e Administração. Editora McGraw-Hill do Brasil, São Paulo - SP (1982)

18. Neves, L.A.P.: Extração de células de dados manuscritos em tabelas. Master's thesis, Pontifícia Universidade Católica do Paraná - PUCPR (1999) 